\title{
Food intake, Healthy Eating Index and Dietary inflammatory index in patients with Psoriatic Arthritis
}

\author{
Beatriz Leite $^{1}$, Melissa Morimoto ${ }^{1}$, Patricia Genaro ${ }^{2}$, Nitin Shivappa ${ }^{3}$, James Hèbert ${ }^{3}$ and \\ Marcelo Pinheiro ${ }^{4}$ \\ ${ }^{1}$ Universidade Federal de Sao Paulo, Sao Paulo, Brazil, \\ ${ }^{2}$ Universidade do Vale do Paraíba, Sao Jose dos Campos, Brazil, \\ ${ }^{3}$ University of South Carolina, South Carolina, USA and \\ ${ }^{4}$ Universidade Federal de Sao Paulo, Sao Paulo, USA
}

\begin{abstract}
Psoriatic Arthritis (PsA) is a chronic inflammatory disease that are associated with multiple comorbidities, particularly metabolic syndrome (MetS), obesity, hypertension and diabetes. These findings brought up a potential link between adiposity and psoriasis/ PsA. Though a healthy diet could improve some aspects related to MetS, as well as painful joints and skin lesions in patients with PsA, the aim of this study is to describe the main particularities related to food intake and nutrient consumption, including the dietary inflammatory index (DII) and the healthy eating index (HEI), in patients with PsA. A total of 97 patients with PsA were included in this cross-sectional study. Food intake was evaluated by using a 3-day food-record, HEI and the DII. Energy was adjusted using the residual method in order to control the effects of energy intake when evaluating micronutrient intake. Weight, waist of circumference, Body Mass Index (BMI) and disease activity (PASI, BSA, BASDAI, DAS28-ESR, DAS28-CRP and MDA) were also evaluated. The inferential analysis included $t$-student test, Pearson's correlation and Tukey's test. Kolmogorov-Smirnov test was used to define normality. Level of significance was set as $\mathrm{p}<0.05$. Patients with PsA had hypercaloric diet and inadequate consumption of sodium, vitamin A, vitamin E, magnesium, zinc and copper when compared to Dietary Reference Intake. The HEI had low score (63.2 \pm 10.2), suggesting that more than $90 \%$ of the patients had inappropriate diet and need nutritional intervention. Moreover, the DII score was high $(+2.48 \pm 0.9)$, highlighting a pro-inflammatory pattern. It was also observed an increase of BMI (mean $30.5 \pm 5.7$ $\left.\mathrm{kg} / \mathrm{m}^{2}\right)$, waist circumference $(103.13 \pm 13.26 \mathrm{~cm})$ and a high prevalence of MetS $(54.6 \%)$. Our data showed patients with PsA had high prevalence of MetS, low quality of food intake, diet inadequacy and a pro-inflammatory pattern, especially regarding antioxidants intake.
\end{abstract}

\section{Conflict of Interest}

There is no conflict of interest 